\title{
Does stroke etiology influence outcome in the posterior circulation? An analysis of 107 consecutive acute basilar occlusion thrombectomies
}

\author{
Roberta K. Sefcik, MD, Daniel A. Tonetti, MD, Shashvat M. Desai, MD, Stephanie M. Casillo, BS, \\ Michael J. Lang, MD, Ashutosh P. Jadhav, MD, PhD, and Bradley A. Gross, MD \\ Department of Neurosurgery, University of Pittsburgh Medical Center, Pittsburgh, Pennsylvania
}

\begin{abstract}
OBJECTIVE Acute basilar artery occlusion (BAO) harbors a more guarded prognosis after thrombectomy compared with anterior circulation large-vessel occlusion. Whether this is a function of a greater proportion of atherosclerotic/ intrinsic lesions is not well studied. The authors aimed to elucidate the prevalence and predictors of intracranial intrinsic atherosclerotic disease in patients with acute BAO and to compare angiographic and clinical outcomes between patients with $\mathrm{BAO}$ secondary to embolic versus intrinsic disease.
\end{abstract}

METHODS A prospectively maintained stroke database was reviewed for all patients presenting between January 2013 and December 2019 to a tertiary care academic comprehensive stroke center with acute, nontandem BAO. Patient data were extracted, subdivided by stroke mechanism and treatment modality (embolic [thrombectomy only] and intrinsic [thrombectomy + stenting]), and angiographic and clinical results were compared.

RESULTS Of 107 patients, 83 (78\%) had embolic occlusions (thrombectomy only) and 24 (22\%) had intrinsic disease (thrombectomy + stenting). There was no significant difference in patient age, presenting National Institutes of Health Stroke Scale score, time to presentation, selected medical comorbidities (hypertension, hyperlipidemia, diabetes, and atrial fibrillation), prior stroke, and posterior circulation Alberta Stroke Program Early CT Score. Patients with intrinsic disease were more likely to be active smokers $(50 \%$ vs $26 \%, p=0.04)$ and more likely to be male $(88 \%$ vs $48 \%, p=0.001)$. Successful recanalization, defined as a modified Thrombolysis in Cerebral Infarction (mTICI) grade of $2 \mathrm{~b}$ or 3 , was achieved in $90 \%$ of patients and did not differ significantly between the embolic versus intrinsic groups $(89 \%$ vs $92 \%, p$ $>0.99$ ). A 90-day good outcome (modified Rankin Scale [mRS] score 0-2) was found in 37\% of patients overall and did not differ significantly between the two groups ( $36 \%$ vs $41 \%, p=0.41$ ). Mortality was $40 \%$ overall and did not significantly differ between groups (41\% vs $36 \%, p=0.45)$.

CONCLUSIONS In the current study, demographic and clinical results for acute BAO showed that compared with intrinsic disease, thromboembolic disease is a more common mechanism of acute $\mathrm{BAO}$, with $78 \%$ of patients undergoing thrombectomy alone. However, there was no significant difference in revascularization and outcome results between patients with embolic disease and those with intrinsic disease.

https://thejns.org/doi/abs/10.3171/2021.4.FOCUS2189

KEYWORDS reperfusion; stroke; thrombectomy; stent; atherosclerosis

$\mathrm{T}$ wo main pathological mechanisms underlie acute basilar artery occlusion (BAO), either an embolic or an intrinsic atherosclerotic thrombosis. ${ }^{1}$ These contrasting pathologies present to clinical attention in a similar manner and can be difficult to differentiate prior to endovascular intervention. Without treatment, acute BAO is a fatal or severely debilitating disease; ${ }^{2}$ nearly $80 \%$ of patients have unfavorable outcomes, with modified Rankin Scale (mRS) scores of 4-6. ${ }^{3}$
Endovascular therapy (EVT) for anterior circulation large-vessel occlusion (LVO) is supported by class 1 evidence. ${ }^{4}$ Despite a lack of class 1 evidence, EVT for BAO is widely practiced and several clinical trials have evaluated the benefit of EVT for BAO in a prospective manner. ${ }^{5-7}$ Given the differences in the underlying mechanism of BAO between embolic and intrinsic atherosclerosis, endovascular management strategies addressing these contrasting pathologies differ. Treatment of residual basilar stenosis after

ABBREVIATIONS BAO = basilar artery occlusion; EVT = endovascular therapy; IV tPA = intravenous tissue plasminogen activator; LVO = large-vessel occlusion; mRS = modified Rankin Scale; $\mathrm{mTICI}=$ modified Thrombolysis in Cerebral Infarction; NIHSS = National Institutes of Health Stroke Scale; pc-ASPECTS = posterior circulation Alberta Stroke Program Early CT Score. 


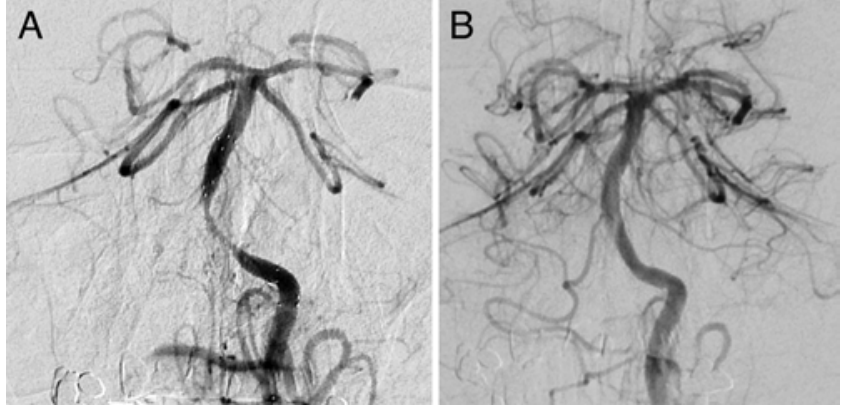

FIG. 1. Anteroposterior view of the left vertebral run with a stentriever deployed, demonstrating the causative intrinsic lesion $(\mathbf{A})$, and the control run poststent (B).

thrombectomy necessitating the deployment of permanent stents with or without angioplasty has been previously described as a treatment for basilar atherosclerosis. ${ }^{8-18}$

It remains unknown whether there are predictors of intrinsic atherosclerotic disease underlying an acute BAO. Knowledge of predictors of intrinsic atherosclerotic disease that requires intracranial stenting for vessel recanalization may allow for hastened reperfusion in patients with acute BAO. The purpose of this study was to 1) elucidate the prevalence and predictors of intracranial intrinsic atherosclerotic disease in patients with acute BAO in a North American cohort, and 2) compare angiographic and clinical outcomes between patients with BAO secondary to embolic versus intrinsic disease.

\section{Methods}

A retrospective analysis of prospectively collected data was performed for all patients presenting to a tertiary care academic comprehensive stroke center with posterior circulation acute ischemic stroke between January 2013 and December 2019. Demographic characteristics, clinical and radiological data, and treatment and procedural information were extracted and analyzed. This study was approved by the local institutional review board.

\section{Patient Selection}

Patients with acute ischemic stroke who presented to a single comprehensive stroke center and underwent thrombectomy for a BAO during the study period were analyzed. Occlusion location was confirmed by CTA, MRA, and/or cerebral angiography. Patients were excluded if the occlusion was distal to the basilar artery (e.g., of the posterior cerebral artery) or in the case of tandem lesions (vertebral ostial occlusion and concomitant BAO).

\section{Procedural Technique}

The manual aspiration thrombectomy procedure was performed at one of two comprehensive stroke centers affiliated with a large academic institution by one of four neurointerventionalists. Briefly, the cervical vasculature (typically $\mathrm{V}_{2}$ ) was catheterized using standard techniques with a long 6-Fr sheath. After an LVO was confirmed via base catheter fluoroscopy, a triaxial system comprising an aspiration catheter, a microcatheter, and a microwire was advanced through the thrombus. The aspiration catheter was advanced to the face of the thrombus, and the microcatheter and wire were removed. Manual aspiration was applied via syringe, which was subsequently withdrawn slowly under continuous aspiration. The 6-Fr long sheath was then aspirated to remove any additional thrombus.

If the basilar artery remained flow limited because of stenosis secondary to a presumed atherosclerotic plaque, a stentriever (Solitaire, Stryker Neurovascular) was deployed over the lesion and a run was performed with the stentriever deployed (Fig. 1). This run was utilized to measure the basilar artery diameters on both sides of the lesion, and an appropriately sized balloon-mounted coronary stent (Multi-Link Vision Coronary Stent System, Abbott Vascular; or Integrity Coronary Stent System, Medtronic) was selected. Patients were administered a weight-based loading dose of intravenous eptifibatide. A 115-cm Catalyst 5 (Stryker Neurovascular) catheter or, in less recent cases, a 115-cm 058 Navien (Medtronic) catheter was guided through the 6-Fr sheath over the microcatheter-microwire to the plaque, and the stent was deployed through the intermediate catheter over the microwire. A poststent run was then performed. After the procedure, patients were administered rectal aspirin on the angiogram table followed by a clopidogrel load once CT had confirmed no hemorrhagic complication. In all cases, a modified Thrombolysis in Cerebral Infarction (mTICI) grade was assigned according to the original TICI classification, ${ }^{19}$ with an mTICI grade of $2 \mathrm{~b}$ representing a reperfusion of at least two-thirds of the vascular territory affected.

\section{Study Variables}

Baseline demographics (age, sex), clinical (stroke severity, time from symptom onset, risk factor/medical history profile) and radiographic (posterior circulation Alberta Stroke Program Early CT Score [pc-ASPECTS], occlusion location) characteristics, adjunctive intravenous tissue plasminogen activator (IV tPA) information, and procedural technique and result (mTICI grade) were collected and analyzed. In addition to mTICI grade, outcome variables included early recovery, early deterioration, postoperative hemorrhage, and outcome as defined by the $\mathrm{mRS}$ score at 90 days.

\section{Stratified Analysis}

Patients were stratified by the procedure required to achieve revascularization. Patients who underwent thrombectomy only were compared with those who required stenting and/or angioplasty due to flow-limiting atherosclerotic disease, and their demographics and outcomes were analyzed and compared, including the revascularization result (mTICI grade) and the clinical outcome (90-day mRS score and mortality).

\section{Statistical Analysis}

Statistical analysis was performed using IBM SPSS Statistics version 23 (IBM Corp.). Univariate analyses were performed using analysis of variance for continuous variables and the Pearson chi-square test for categorical variables. Multivariate logistic regression analysis was 
TABLE 1. Overall baseline characteristics

\begin{tabular}{lcccc}
\hline & All Patients & Thrombectomy Only & Thrombectomy + Stenting & $p$ Value \\
\hline No. of patients & 107 & 83 & 24 & \\
\hline Mean age, yrs & $64.5 \pm 16.8$ & $65.3 \pm 17.3$ & $62.4 \pm 14.8$ & 0.479 \\
\hline Male sex & $61(57)$ & $40(48.2)$ & $21(87.5)$ & 0.001 \\
\hline Mean NIHSS score & $18.7 \pm 9.3$ & $19.1 \pm 9.2$ & $17.3 \pm 9.6$ & 0.416 \\
\hline Mean last known well to arrival, hrs & $13.5 \pm 17.3$ & $12.1 \pm 16.1$ & $18.6 \pm 20.4$ & 0.138 \\
\hline Hypertension & $80 / 103(77.7)$ & $61 / 79(77.2)$ & $19 / 24(79.2)$ & $>0.99$ \\
\hline Hyperlipidemia & $48 / 103(46.6)$ & $36 / 79(45.6)$ & $12 / 24(50)$ & 0.816 \\
\hline Diabetes mellitus & $24 / 98(24.5)$ & $20 / 76(26.3)$ & $4 / 22(18.2)$ & 0.577 \\
\hline Atrial fibrillation & $28 / 99(28.3)$ & $24 / 78(30.8)$ & $4 / 21(19)$ & 0.415 \\
\hline Smoking & $31 / 100(31)$ & $20 / 78(25.6)$ & $11 / 22(50)$ & 0.038 \\
\hline Prior stroke & $22 / 96(22.9)$ & $15 / 75(20)$ & $7 / 21(33.3)$ & 0.242 \\
\hline Mean pc-ASPECTS & $9 \pm 1.3$ & $9.1 \pm 1.2$ & $8.7 \pm 1.5$ & 0.157 \\
\hline IV tPA & $18 / 99(18.2)$ & $15 / 77(19.5)$ & $3 / 22(13.6)$ & 0.756 \\
\hline
\end{tabular}

Values represent the number of patients (\%) unless stated otherwise. Mean values are presented as the mean \pm SD.

subsequently performed to identify independent predictors of 90-day outcomes. Outcome measures, including final reperfusion, mRS score at 90 days, mortality, and complications, were directly compared using the Pearson chi-square test.

\section{Results}

\section{Patient Demographics}

A total of 107 patients with acute ischemic stroke due to nontandem BAO underwent endovascular treatment during the study period. Five additional patients with tandem vertebral ostial disease were excluded from the analysis. Eighty-three patients (78\%) underwent thrombectomy only, and 24 patients (22\%) underwent thrombectomy in combination with basilar artery stenting during the study period. The mean patient age was 64.5 years with a standard deviation \pm 16.8 years, and $57 \%$ of patients were male (Table 1). The mean National Institutes of Health Stroke Scale (NIHSS) score at hospital presentation was $18.7 \pm$ 9.3. The mean pc-ASPECTS was $9.0 \pm 1.3$, and $18.2 \%$ of patients received IV tPA.

Compared with patients treated with thrombectomy alone, those requiring stenting were more likely to be male $(88 \%$ vs $48 \%, \mathrm{p}=0.001)$ and were more frequently tobacco smokers $(50 \%$ vs $26 \%, \mathrm{p}=0.038)$. There was otherwise no significant difference between the two patient groups in age, presenting NIHSS score, when the patient was last known to be well, medical comorbidities, pc-ASPECTS, or IV tPA administration.

\section{Outcomes}

An mTICI reperfusion grade $\geq 2 \mathrm{~b}$ was achieved in $90 \%$ of patients overall; this was not significantly different between the groups (Table 2). Fifty-one percent of the patients had early clinical recovery, and $9 \%$ experienced deterioration; these rates did not significantly differ between the two groups. Postprocedural hemorrhage was seen in $6 \%$ of patients, which also did not significantly differ between the two groups. There were no cases of acute stent reocclusion. Overall, an mRS score of 0-2 at 90 days was achieved in $37 \%$ of patients and $40 \%$ of patients died, with no significant difference in either outcome between the two groups.

Multivariate analysis was performed to determine predictors of outcome. After controlling for male sex, time to presentation, smoking status, pc-ASPECTS, and stent use, there were no independent predictors of functional outcome (mRS score $0-2)$ at 90 days or 90 -day mortality.

\section{Discussion}

The guarded prognosis for acute vertebrobasilar oc-

TABLE 2. Angiographic and clinical outcomes

\begin{tabular}{lcccc}
\hline & All Patients & Thrombectomy Only & Thrombectomy + Stenting & $p$ Value \\
\hline mTICl grade $\geq 2 \mathrm{~b}$ & $96 / 107(89.7)$ & $74 / 83(89.2)$ & $22 / 24(91.7)$ & $>0.99$ \\
\hline Early clinical recovery & $53 / 104(51)$ & $44 / 83(53)$ & $9 / 21(42.9)$ & 0.469 \\
\hline Hemorrhage on 24-hr CT & $6 / 107(5.6)$ & $4 / 83(4.8)$ & $2 / 24(8.3)$ & 0.614 \\
\hline Early neurological deterioration & $9 / 104(8.7)$ & $7 / 83(8.4)$ & $2 / 21(9.5)$ & $>0.99$ \\
\hline mRS score 0-2 at 90 days & $36 / 98(36.7)$ & $27 / 76(35.5)$ & $9 / 22(40.9)$ & 0.412 \\
\hline Mortality & $39 / 98(39.8)$ & $31 / 76(40.8)$ & $8 / 22(36.4)$ & 0.454 \\
\hline
\end{tabular}

Values represent the number of patients (\%) unless stated otherwise. 
clusion $^{2}$ is worthy of more careful scrutiny. The finding that intrinsic disease underlies acute BAO in one-fifth of North American patients in this study begs the question of whether this is a contributing factor to worse outcomes given the added need to address the underlying lesion in addition to a standard thrombectomy.

Systematic study of vertebrobasilar thrombectomy is less prevalent in the literature compared with the more common anterior circulation LVOs. The BEST study was a trial conducted at 28 stroke centers in China that attempted to randomize patients presenting within 8 hours of symptom onset with acute vertebrobasilar occlusion who underwent EVT plus medical therapy or medical therapy alone. ${ }^{7}$ The trial was terminated early due to high crossover rates and poor recruitment. Good outcome at 90 days (mRS score $0-3$ ) was seen in $42 \%$ of patients in the intervention group and $32 \%$ in the control group, a difference not meeting statistical significance. However, a secondary, as-treated analysis demonstrated statistically significantly greater rates of good outcome in patients who underwent endovascular treatment $(47 \%)$ versus those assigned to medical therapy without crossover (24\%).

\section{Key Results}

Our results demonstrate a good overall rate $(90 \%)$ of successful reperfusion in patients with acute BAO (mTICI grade $\geq 2 b$ ), comparable to success rates in recent multicenter data and other intrinsic series. ${ }^{20,25}$ Notably, occlusion etiology did not influence this rate significantly. More supple intermediate catheters have made the navigation of noncompliant stents to desired intracranial target vessels far more facile, ${ }^{21}$ explaining the reasonable technical success for this approach in our study with 24 patients who underwent acute stenting. Of note, potential predictors for intrinsic lesions included male sex and smoking status.

As successful reperfusion did not significantly differ between the two patient groups, rates of 90-day good outcome, defined as an mRS score of 0-2 at 90 days, and mortality did not significantly differ either. These results are not entirely dependent, as differing stroke etiology may serve as a biomarker/predictor of overall outcome independent of reperfusion result. Periprocedural complications, particularly hemorrhagic complications, did not significantly differ between the two groups; this is a relevant finding in the context of a ubiquitous dual antiplatelet load in the intrinsic disease population and will require substantiating across a larger patient cohort. While some may advocate for administration of a glycoprotein IIb/IIIA inhibitor in lieu of intracranial stenting for acute occlusion due to intrinsic disease, ${ }^{22}$ the concern for an incompletely treated intrinsic lesion bares the intuitive prospective risk of recurrent transient ischemic attack or stroke; extrapolating from the SAMMPRIS trial, ${ }^{23}$ this rate was $6 \%$ at 30 days.

\section{Limitations}

While our study was a prospective analysis of consecutive patients with blinded data review and good retention for 90-day follow-up, we are limited by a relatively small sample size of patients who underwent acute stenting, lim- iting our analysis of complications. In addition, a lack of a statistically significant difference in reperfusion and good outcome rates may be overcome by a larger sample size. TICI recanalization grades were not blinded but rather assigned by the interventionalist posttreatment. Future multicenter analyses with blinded TICI grading would improve the robustness of this initial analysis of acute BAO in a North American population. Furthermore, patients were defined as having intrinsic or embolic disease by the revascularization procedure employed. A pretreatment radiographic definition would be superior.

\section{Interpretation and Generalizability}

Our results demonstrate that demographic factors may be useful in predicting the likelihood of an intrinsic lesion (male sex, smoking status). Furthermore, in contrast to the BEST trial, 7 our North American population harbored an approximately $20 \%$ prevalence of intrinsic disease underlying acute $\mathrm{BAO}$, reinforcing, as is known, that different populations harbor differing frequencies of underlying atherosclerotic occlusion; this limits the external validity of our results to these differing populations.

Our results also suggest that reperfusion is similarly feasible and safe in patients with athero-occlusive lesions with the usage of noncompliant coronary stents as compared with more typical thromboembolic occlusions requiring thrombectomy alone. Interestingly, clinical outcome results were fairly uniform across both cohorts as well. Our results differ from the current body of literature comparing posterior circulation lesions in which patients with intrinsic disease have lower recanalization rates. ${ }^{24,25}$

\section{Conclusions}

Thromboembolic disease was a more common mechanism of acute BAO than intrinsic disease, with $78 \%$ of patients in the current study undergoing thrombectomy alone. Intrinsic disease was seen more often in active smokers and males. Rates of successful recanalization and functional outcomes were comparable between patients with and without intrinsic disease.

\section{References}

1. Gao F, Lo WT, Sun X, et al. Combined use of mechanical thrombectomy with angioplasty and stenting for acute basilar occlusions with underlying severe intracranial vertebrobasilar stenosis: preliminary experience from a single Chinese center. AJNR Am J Neuroradiol. 2015;36(10):1947-1952.

2. Hacke $\mathrm{W}$, Zeumer $\mathrm{H}$, Ferbert $\mathrm{A}$, et al. Intra-arterial thrombolytic therapy improves outcome in patients with acute vertebrobasilar occlusive disease. Stroke. 1988;19(10):1216-1222.

3. Schonewille WJ, Algra A, Serena J, et al. Outcome in patients with basilar artery occlusion treated conventionally. $J$ Neurol Neurosurg Psychiatry. 2005;76(9):1238-1241.

4. Goyal M, Menon BK, van Zwam WH, et al. Endovascular thrombectomy after large-vessel ischaemic stroke: a metaanalysis of individual patient data from five randomised trials. Lancet. 2016;387(10029):1723-1731.

5. Schonewille WJ, Wijman CAC, Michel P, et al. Treatment and outcomes of acute basilar artery occlusion in the Basilar Artery International Cooperation Study (BASICS): a prospective registry study. Lancet Neurol. 2009;8(8):724-730.

6. Zi W, Qiu Z, Wu D, et al. Assessment of endovascular treat- 
ment for acute basilar artery occlusion via a nationwide prospective registry. JAMA Neurol. 2020;77(5):561-573.

7. Liu X, Dai Q, Ye R, et al. Endovascular treatment versus standard medical treatment for vertebrobasilar artery occlusion (BEST): an open-label, randomised controlled trial. Lancet Neurol. 2020;19(2):115-122.

8. Nardai S, Kis B, Gubucz I, et al. Coronary stent implantation for acute basilar artery occlusion with underlying stenosis. EuroIntervention. 2020;16(12):e1021-e1028.

9. Andersson T, Kuntze Söderqvist Å, Söderman M, et al. Mechanical thrombectomy as the primary treatment for acute basilar artery occlusion: experience from 5 years of practice. J Neurointerv Surg. 2013;5(3):221-225.

10. Giorgianni A, Biraschi F, Piano M, et al. Endovascular treatment of acute basilar artery occlusion: Registro Endovascolare Lombardo Occlusione Basilar Artery (RELOBA) Study Group experience. J Stroke Cerebrovasc Dis. 2018;27(9): 2367-2374.

11. Mourand I, Machi P, Milhaud D, et al. Mechanical thrombectomy with the Solitaire device in acute basilar artery occlusion. J Neurointerv Surg. 2014;6(3):200-204.

12. Roth C, Mielke A, Siekmann R, Ferbert A. First experiences with a new device for mechanical thrombectomy in acute basilar artery occlusion. Cerebrovasc Dis. 2011;32(1):28-34.

13. Lin YH, Chen KW, Tang SC, Lee CW. Endovascular treatment outcome and CT angiography findings in acute basilar artery occlusion with and without underlying intracranial atherosclerotic stenosis. J Vasc Interv Radiol. 2020;31(5): 747-753.

14. Sun X, Tong X, Gao F, et al. Endovascular treatment for acute basilar artery occlusion: a single center retrospective observational study. BMC Neurol. 2019;19(1):315.

15. Lee YY, Yoon W, Kim SK, et al. Acute basilar artery occlusion: differences in characteristics and outcomes after endovascular therapy between patients with and without underlying severe atherosclerotic stenosis. AJNR Am J Neuroradiol. 2017;38(8):1600-1604.

16. Mordasini P, Brekenfeld C, Byrne JV, et al. Technical feasibility and application of mechanical thrombectomy with the Solitaire FR Revascularization Device in acute basilar artery occlusion. AJNR Am J Neuroradiol. 2013;34(1):159-163.

17. Espinosa de Rueda M, Parrilla G, Zamarro J, et al. Treatment of acute vertebrobasilar occlusion using thrombectomy with stent retrievers: initial experience with 18 patients. AJNR Am J Neuroradiol. 2013;34(5):1044-1048.

18. Siebert E, Bohner G, Zweynert S, et al. Revascularization techniques for acute basilar artery occlusion: technical considerations and outcome in the setting of severe posterior circulation steno-occlusive disease. Clin Neuroradiol. 2019; 29(3):435-443.

19. Higashida RT, Furlan AJ, Roberts H, et al. Trial design and reporting standards for intra-arterial cerebral thrombolysis for acute ischemic stroke. Stroke. 2003;34(8):e109-e137.
20. Brinjikji W, Raz E, De Leacy R, et al. MRS SOFIA: a multicenter retrospective study for use of Sofia for revascularization of acute ischemic stroke. J Neurointerv Surg. Published online February 1, 2021. doi:10.1136/neurintsurg-2020-017042

21. Gross BA, Desai SM, Walker G, et al. Balloon-mounted stents for acute intracranial large vessel occlusion secondary to presumed atherosclerotic disease: evolution in an era of supple intermediate catheters. J Neurointerv Surg. 2019; 11(10):975-978.

22. Kang DH, Yoon W, Kim SK, et al. Endovascular treatment for emergent large vessel occlusion due to severe intracranial atherosclerotic stenosis. J Neurosurg. 2019;130(6):1949_ 1956.

23. Chimowitz MI, Lynn MJ, Derdeyn CP, et al. Stenting versus aggressive medical therapy for intracranial arterial stenosis. N Engl J Med. 2011;365(11):993-1003.

24. Baik SH, Park HJ, Kim JH, et al. Mechanical thrombectomy in subtypes of basilar artery occlusion: relationship to recanalization rate and clinical outcome. Radiology. 2019;291(3): 730-737.

25. Zhang X, Luo G, Jia B, et al. Differences in characteristics and outcomes after endovascular therapy: a single-center analysis of patients with vertebrobasilar occlusion due to underlying intracranial atherosclerosis disease and embolism. Interv Neuroradiol. 2019;25(3):254-260.

\section{Disclosures}

Dr. Gross is a consultant for Medtronic and MicroVention.

\section{Author Contributions}

Conception and design: Gross, Jadhav. Acquisition of data: Gross, Sefcik, Tonetti, Desai, Casillo. Analysis and interpretation of data: Gross, Sefcik, Tonetti, Desai, Casillo. Drafting the article: Gross, Sefcik, Tonetti. Critically revising the article: all authors. Reviewed submitted version of manuscript: all authors. Approved the final version of the manuscript on behalf of all authors:

Gross. Statistical analysis: Desai. Study supervision: Gross, Lang, Jadhav.

\section{Supplemental Information}

\section{Abstract Presentations}

Portions of this study were accepted for presentation at the 2020 CNS Annual Meeting, Miami, Florida.

\section{Correspondence}

Bradley A. Gross: University of Pittsburgh Medical Center, Pittsburgh,PA.grossb2@upmc.edu. 